\title{
Clinical and pathologic features of ductal carcinoma in situ associated with the presence of flat epithelial atypia: an analysis of 543 patients
}

Laura C Collins ${ }^{1}$, Ninah A Achacoso ${ }^{2}$, Larissa Nekhlyudov ${ }^{3}$, Suzanne W Fletcher ${ }^{3}$, Reina Haque ${ }^{4}$, Charles P Quesenberry Jr², Najeeb S Alshak ${ }^{5}$, Balaram Puligandla ${ }^{6,7}$, Gilbert L Brodsky ${ }^{8}$, Stuart J Schnitt ${ }^{1}$ and Laurel A Habel ${ }^{2,9}$

${ }^{1}$ Department of Pathology, Beth Israel Deaconess Medical Center, Harvard Medical School, Boston, MA, USA; ${ }^{2}$ Division of Research, Kaiser Permanente Northern California, Oakland, CA, USA; ${ }^{3}$ Department of Ambulatory Care and Prevention, Harvard Medical School, Harvard Pilgrim Health Care, Boston, MA, USA; ${ }^{4}$ Department of Research and Evaluation, Kaiser Permanente Southern California, Pasadena, CA, USA; ${ }^{5}$ Department of Pathology, Kaiser Permanente Medical Center, Los Angeles, CA, USA; ${ }^{6}$ Department of Pathology, Kaiser Permanente, Oakland Medical Center, Oakland, CA, USA; ${ }^{7}$ Department of Pathology, University of California, Berkeley, CA, USA; ${ }^{8}$ Department of Pathology, Harvard Vanguard Medical Associates, Boston, MA, USA and ${ }^{9}$ Department of Health and Research Policy, Stanford University School of Medicine, Stanford, CA, USA

Flat epithelial atypia is an alteration of mammary terminal duct lobular units that is considered to be a precursor to, or early stage in, the development of some forms of ductal carcinoma in situ. No prior study has systematically evaluated the relationship between various clinico-pathologic features of ductal carcinoma in situ and the presence of coexistent flat epithelial atypia. An understanding of such relationships could provide insight into the connection between flat epithelial atypia and ductal carcinoma in situ. We reviewed slides from 543 ductal carcinoma in situ patients enrolled in a case-control study assessing epidemiologic and pathologic risk factors for local recurrence. We examined the association between the presence of flat epithelial atypia and various clinical factors, pathologic features of the ductal carcinoma in situ, and the presence of coexistent atypical ductal hyperplasia, lobular neoplasia, and non-atypical columnar cell lesions. In univariate analysis, the presence of flat epithelial atypia was significantly related to ductal carcinoma in situ nuclear grade (most common in low grade, least common in high grade; $P<0.0001$ ), architectural pattern (most common in micropapillary and cribriform, least common in comedo; $P<0.0001)$, absence of comedo necrosis $(P<0.001)$, absence of stromal desmoplasia $(P=0.02)$ and absence of stromal inflammation $(P=0.03)$. In multivariable analysis, features of ductal carcinoma in situ independently associated with flat epithelial atypia were micropapillary and cribriform patterns and absence of comedo necrosis. Additionally, flat epithelial atypia was significantly associated with the presence of atypical ductal hyperplasia, lobular neoplasia, and columnar cell lesions in both univariate and multivariable analyses. These observations provide support for a precursorproduct relationship between flat epithelial atypia and ductal carcinoma in situ lesions that exhibit particular features such as micropapillary and cribriform patterns and absence of comedo necrosis.

Modern Pathology (2007) 20, 1149-1155; doi:10.1038/modpathol.3800949; published online 31 August 2007

Keywords: flat epithelial atypia; ductal carcinoma in situ; breast cancer

Flat epithelial atypia is an alteration of mammary terminal duct lobular units in which the native

Correspondence: Dr LC Collins, MD, Department of Pathology, Beth Israel Deaconess Medical Center, Harvard Medical School, 330 Brookline Avenue, Boston, MA 02215, USA.

E-mail: lcollins@bidmc.harvard.edu

Received 16 June 2007; revised 10 July 2007; accepted 16 July 2007; published online 31 August 2007 epithelial cells are replaced by one to several layers of cuboidal to columnar epithelial cells that show cytologic atypia, most commonly of the low grade or monomorphic type resembling that seen in lowgrade ductal carcinoma in situ. While the term 'flat epithelial atypia' was first introduced by the World Health Organization Working Group on the Pathology and Genetics of Tumors of the Breast in 2003, ${ }^{1}$ this lesion has been recognized for many years 
under a variety of different names, most notably 'clinging carcinoma' of the monomorphic type. ${ }^{2,3}$

It has been difficult to assess the clinical significance of flat epithelial atypia due to variations in the terminology used in the past and the limited number of cases that have been studied in a systematic fashion. Nonetheless, several small observational studies have clearly shown that the lesion now recognized as flat epithelial atypia commonly coexists with well-developed examples of atypical ductal hyperplasia, low-grade ductal carcinoma in situ and tubular carcinoma, and that the cells comprising the flat epithelial atypia share cytologic and immunophenotypic features with the cells comprising these other lesions. ${ }^{3-14}$ A number of previous studies have also noted an association between flat epithelial atypia and lobular neoplasia (lobular carcinoma in situ and atypical lobular hyperplasia). ${ }^{11,13-15}$

Recent studies have begun to investigate the genetic alterations in flat epithelial atypia through loss of heterozygosity, comparative genomic hybridization, and $\mathrm{X}$ chromosome inactivation assays. ${ }^{12,16,17}$ Although these studies were not prospective, were based on small sample sizes, or included a select group of patients, they have demonstrated a number of genetic similarities in flat epithelial atypia and coexistent ductal carcinoma in situ and invasive cancer, implying an evolutionary relationship.

Based on the aforementioned observations, flat epithelial atypia appears to be a neoplastic proliferation that may well represent either a precursor to, or the earliest morphologic manifestation of, low-grade ductal carcinoma in situ, as well as a precursor to invasive carcinoma, particularly tubular carcinoma. However, no prior study has systematically evaluated the relationships between various clinico-pathologic features of ductal carcinoma in situ and the presence of coexistent flat epithelial atypia. The goal of the present study was to examine the connection between flat epithelial atypia and ductal carcinoma in situ among women enrolled in a population-based study.

\section{Materials and methods}

\section{Study Population}

The population for this study consists of patients derived from a case-control study nested within a cohort of women diagnosed with a first primary unilateral ductal carcinoma in situ and treated with breast-conserving therapy between 1990 and 2001 and for whom pathology review has been completed to date. The cohort was identified using cancer registries or electronic medical records at three health plans which are members of the Cancer Research Network, a network of research programs, enrollee populations, and databases of 11 health maintenance organization members whose overall goal is to conduct collaborative research to determine the effectiveness of preventive, curative, and supportive interventions for major cancers. The three health plans participating in this study were Kaiser Permanente of Northern California, Kaiser Permanente of Southern California and Harvard Pilgrim Health Care.

Patients were eligible if they were less than 85 years at diagnosis and had no prior breast cancer or invasive cancer at another site. Patients were excluded if breast cancer (ductal carcinoma in situ or invasive disease) had been diagnosed in the contralateral breast at the time of the index ductal carcinoma in situ diagnosis or if they had a mastectomy within 6 months of their ductal carcinoma in situ diagnosis. Patients were also excluded from this analysis if the pathology review determined that the initial diagnosis was not ductal carcinoma in situ (see below).

At each of the three health plans, medical records of potentially eligible patients were reviewed to confirm the initial diagnosis, treatment and laterality of the index ductal carcinoma in situ and to obtain information on subsequent breast cancer events. Information was also collected on surveillance mammography, and on all subsequent breast biopsies. In addition, data were abstracted on several patients and clinical factors at the time of their index ductal carcinoma in situ (eg, use of exogenous hormones [including tamoxifen], demographics, reproductive history), as well as on several patient and clinical factors after their index ductal carcinoma in situ.

The cohort was followed from the initial ductal carcinoma in situ diagnosis until the earliest of the following events: subsequent ductal carcinoma in situ or invasive breast cancer, mastectomy of the ipsilateral breast, death, termination of health plan membership, or end of study period (last chart note at time of medical record review).

\section{Design of Nested Case-Control Study}

Cases were patients whose first event during followup was a breast cancer recurrence. A recurrence was defined as any ipsilateral breast cancer event (ductal carcinoma in situ or invasive) or any regional or distant metastasis. At the time of each case's recurrence, up to two controls were randomly selected from all surviving patients with no evidence of a breast cancer recurrence as of that date (ie, incidence density sampling). Controls were individually matched to their case on age ( $<45,45-54,55-64,65-84$ years), calendar year of diagnosis (1990-1991, 1992-1993, 1994-1995, 1996-1997, 1998-1999 and 2000-2001), and health plan.

A total of 3668 potentially eligible ductal carcinoma in situ patients were identified. Of these, 517 were ineligible for one or more of the following 
reasons: miscoding of ductal carcinoma in situ in the tumor registry $(n=97)$, prior breast or other cancer $(n=216)$, bilateral breast cancer at diagnosis $(n=29)$, treatment of index ductal carcinoma in situ with mastectomy $(n=96), 85$ years of age or older at diagnosis $(n=15)$, not followed within the health plan for at least 6 months $(n=98)$. In addition, medical records were unavailable on 82 patients. Of the 3069 remaining patients, we identified 343 with a recurrence (cases) and selected 603 controls. Diagnostic slides were unavailable on 31 cases and 79 controls. Pathology review to date has been completed on 598 patients. Of these, 21 cases and 34 controls were found not to be ductal carcinoma in situ at pathology review, leaving 543 patients (214 cases and 329 controls) for the present analysis. When a control was found not to be ductal carcinoma in situ on pathology review or diagnostic slides were unavailable $(n=34)$, another control was randomly selected.

\section{Pathology Review}

Available histologic slides and pathology reports from all biopsy and surgical procedures (core needle biopsy, initial excision and all re-excisions) pertaining to the index ductal carcinoma in situ diagnosis were obtained and reviewed simultaneously by two breast pathologists (LCC, SJS) blinded to the casecontrol status of the patient. Information regarding specimen size, presence of a macroscopically evident tumor, macroscopic tumor size (if present), status of the surgical margins, and the proportion of the specimen submitted was abstracted from pathology reports. Histologic features of ductal carcinoma in situ evaluated included architectural patterns (comedo, solid, cribriform, micropapillary, papillary, or clinging), nuclear grade (low, intermediate, or high), comedo necrosis, involvement of lobules (defined as the presence of ductal carcinoma cells within identifiable, pre-existing lobular units), stromal desmoplasia, stromal inflammation, and status of surgical margins. For architectural pattern the primary (or predominant), secondary and tertiary patterns were recorded; for the purposes of this analysis only the predominant architectural pattern was used. Similarly, for nuclear grade the predominant as well as the highest nuclear grade was recorded; for the purposes of this analysis, the predominant nuclear grade was used. The presence in breast tissue adjacent to ductal carcinoma in situ of atypical ductal hyperplasia (either immediately adjacent or as separate foci), lobular carcinoma in situ, atypical lobular hyperplasia, flat epithelial atypia and non-atypical columnar cell lesions (columnar cell change and columnar cell hyperplasia) as well as other benign non-proliferative and proliferative changes was also recorded.

As described previously, ${ }^{18}$ flat epithelial atypia is characterized by enlarged terminal duct lobular

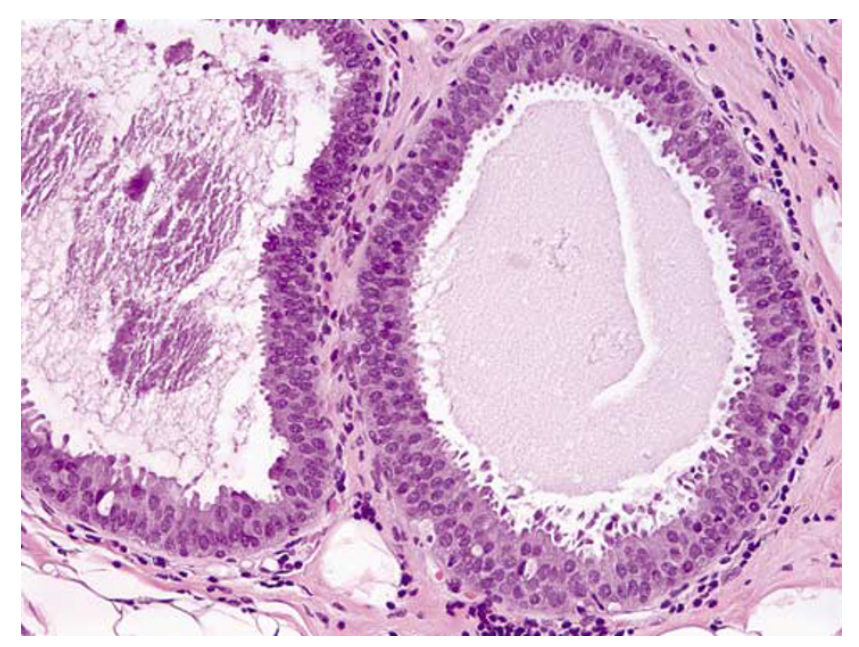

Figure 1 Flat epithelial atypia. The acini in this terminal duct lobular unit are enlarged and dilated; the lumina contain secretions and calcifications. The epithelial lining consists of columnar cells with prominent apical snouts. The nuclei are round to ovoid and monomorphic.

units with variably dilated acini, which often contain flocculent secretory material and calcifications. The epithelial lining of the acini consists of one to several layers of cuboidal to columnar cells that exhibit monomorphic-type cytologic atypia with round, regular nuclei (Figure 1). While these cells can show some cellular stratification and tufting, flat epithelial atypia lacks complex architectural patterns such as well-developed micropapillations, rigid cellular bridges, bars and arcades, or punched-out fenestrations. Lesions that show both monomorphic cytologic atypia and complex architectural patterns as described above are best considered atypical ductal hyperplasia or ductal carcinoma in situ, depending upon the severity and extent of the cytologic and architectural features. The relatively round, monomorphic appearance of the nuclei distinguishes flat epithelial atypia from non-atypical columnar cell lesions (columnar cell change and columnar cell hyperplasia) which are characterized by ovoid to elongated nuclei that are regularly oriented perpendicular to the basement membrane of the involved spaces. ${ }^{18}$ Lesions with a flat growth pattern but with high-grade cytologic atypia were classified as ductal carcinoma in situ with a 'clinging' pattern and not as flat epithelial atypia.

\section{Statistical Analysis}

For the current study, we conducted an interim analysis of all eligible patients for whom pathology review has been completed to date. We examined the clinical and pathologic features of ductal carcinoma in situ associated with the presence of flat epithelial atypia regardless of the patient's case- 
control status. The $\chi^{2}$ test was used to examine whether flat epithelial atypia was statistically significantly associated with other variables of interest without stratification by case-control status. Logistic regression modeling was used to examine which variables were associated with flat epithelial atypia, independent of their association with other variables. Variables of interest included: age, method of detection of ductal carcinoma in situ, history of breast cancer in first-degree blood relative, nuclear grade, architectural pattern, presence of necrosis, and other associated pathologic changes (ie, atypical ductal hyperplasia, lobular neoplasia, and nonatypical columnar cell lesions).

\section{Institutional Review Board Approval}

The study was approved by the Kaiser Permanente Inter-regional Institutional Review Board and by the Institutional Review Boards at Harvard Pilgrim Health Center and Beth Israel Deaconess Medical Center, Boston, MA, USA.

\section{Results}

Among the 543 women in this analysis, the median patient age was 57 years (range 26-84 years). Ductal carcinoma in situ was diagnosed on a screening mammogram in 424 women (78\%) and because of a palpable mass or other sign or symptom in 115 $(21 \%)$. In the remaining four, the mode of presentation was unknown. The median number of slides reviewed per patient was 18 (range, 1-91 slides).

Overall, flat epithelial atypia was present in 103 $(19 \%)$ of the 543 patients with ductal carcinoma in situ. The prevalence of flat epithelial atypia in association with ductal carcinoma in situ among cases and controls was similar (20 and 19\%, respectively). The presence of flat epithelial atypia did not differ across age at diagnosis, family history of breast cancer, and mode of presentation (Table 1).

In univariate analysis, the presence of flat epithelial atypia was significantly associated with ductal carcinoma in situ nuclear grade (flat epithelial atypia present in 34,23 , and $9 \%$ of cases with low, intermediate, and high nuclear grade, respectively; $P<0.0001$ ), architectural pattern (flat epithelial atypia present in $39,27,15,11$, and $6 \%$ of cases with micropapillary, cribriform, papillary, solid, and comedo patterns, respectively, $P<0.0001$ ), absence of comedo necrosis $(P<0.0004)$, absence of stromal desmoplasia $(P=0.02)$ and absence of stromal inflammation $(P=0.03)$ (Table 2).

In multivariable analysis, pathologic features of ductal carcinoma in situ independently associated with flat epithelial atypia were architectural patterns $(P=0.0006)$ and absence of comedo necrosis $(P=0.005)$. In addition, flat epithelial atypia was associated with the presence of atypical ductal hyperplasia, lobular neoplasia, and non-atypical
Table 1 Relationship between flat epithelial atypia and clinical features of patients with ductal carcinoma in situ

\begin{tabular}{|c|c|c|c|}
\hline & $\mathrm{N}$ & $\begin{array}{l}\text { Flat epithelial } \\
\text { atypia present }\end{array}$ & $\mathrm{P}$-value \\
\hline \multicolumn{4}{|l|}{ Age at diagnosis } \\
\hline$<50$ & 166 & $36(22 \%)$ & 0.2 \\
\hline$>50$ & 377 & $67(18 \%)$ & \\
\hline \multicolumn{4}{|l|}{ Presentation } \\
\hline $\begin{array}{l}\text { Mammographic } \\
\text { abnormality }\end{array}$ & 424 & $77(18 \%)$ & 0.5 \\
\hline $\begin{array}{l}\text { Signs, symptoms } \\
\text { (palpable mass) }\end{array}$ & 115 & $24(21 \%)$ & \\
\hline Unknown & 4 & 2 & \\
\hline \multicolumn{4}{|l|}{ Family history ${ }^{\mathrm{a}}$} \\
\hline No & 430 & $79(18 \%)$ & 0.5 \\
\hline Yes & 97 & $21(22 \%)$ & \\
\hline Unknown & 16 & 3 & \\
\hline
\end{tabular}

${ }^{\mathrm{a}}$ History of breast cancer in first degree blood relative (mother, sister or daughter) noted at or within 6 months of ductal carcinoma in situ diagnosis.

Table 2 Relationship between flat epithelial atypia and pathologic features of ductal carcinoma in situ

\begin{tabular}{lrcc}
\hline $\begin{array}{l}\text { Ductal carcinoma in } \\
\text { situ nuclear grade }\end{array}$ & $\mathrm{N}$ & $\begin{array}{c}\text { Flat epithelial } \\
\text { atypia present }\end{array}$ & P-value \\
\hline Low & 47 & $16(34 \%)$ & $<0.0001$ \\
Intermediate & 301 & $70(23 \%)$ & \\
High & 195 & $17(9 \%)$ & \\
Ductal carcinoma in situ pattern & & & \\
Micropapillary & 36 & $14(39 \%)$ & $<0.0001$ \\
Cribriform & 185 & $50(27 \%)$ & \\
Papillary & 66 & $10(15 \%)$ & \\
Solid & 187 & $21(11 \%)$ & \\
Comedo & 63 & $4(6 \%)$ & \\
Other: clinging & 6 & 4 & \\
Comedo necrosis & & & \\
Absent & 242 & $62(26 \%)$ & 0.0004 \\
Present & 301 & $41(14 \%)$ & \\
Unknown & 4 & 1 & \\
& & & \\
Cancerization of lobules & & $38(18 \%)$ & 0.8 \\
Absent & 205 & $65(19 \%)$ & \\
Present & 337 & 0.03 & \\
Unknown & 1 & & \\
Stromal desmoplasia & & & \\
Absent & 339 & $75(22 \%)$ & 0.02 \\
Present & 204 & $28(14 \%)$ & \\
& & & \\
Stromal inflammation & & & \\
Absent & 368 & $79(22 \%)$ & \\
Present & 175 & $24 \%)$ & \\
\hline
\end{tabular}

${ }^{\mathrm{a}}$ Predominant.

columnar cell lesions in these specimens in both univariate and multivariable analyses $(P<0.005)$ (Tables 3 and 4). 
Table 3 Relationship between flat epithelial atypia, atypical ductal hyperplasia, lobular neoplasia (atypical lobular hyperplasia/lobular carcinoma in situ) and columnar cell change

\begin{tabular}{lccc}
\hline & $\mathrm{N}$ & $\begin{array}{c}\text { Flat epithelial } \\
\text { atypia present } \\
(\%)\end{array}$ & P-value \\
& & & \\
\hline $\begin{array}{l}\text { Atypical ductal hyperplasia } \\
\text { Absent }\end{array}$ & 346 & $37(11)$ & $<0.0001$ \\
Present & 197 & $66(34)$ & \\
Atypical lobular hyperplasia/lobular carcinoma in situ \\
Absent \\
$\begin{array}{l}\text { Present } \\
\text { Columnar cell change } \\
\text { Absent }\end{array}$ & 139 & $59(15)$ & $<0.0001$ \\
Present & 381 & $44(32)$ & \\
& 162 & $53(14)$ & $<0.0001$ \\
\end{tabular}

Table 4 Pathologic features significantly associated with the presence of flat epithelial atypia on multivariable analysis ${ }^{\mathrm{a}}$

\begin{tabular}{lccc}
\hline & $\begin{array}{c}\text { Odds } \\
\text { ratio }\end{array}$ & $\begin{array}{c}95 \% \\
\text { Confidence } \\
\text { interval }\end{array}$ & P-value \\
\hline Architectural pattern & 1.0 & Referent & \\
$\quad$ Solid & 5.1 & $(2.1-12.5)$ & 0.0006 \\
Micropapillary & 2.2 & $(1.2-4.1)$ & \\
Cribriform & 1.0 & $(0.4-2.4)$ & \\
Papillary & 0.7 & $(0.2-2.3)$ & \\
Comedo & & & \\
Necrosis & 1.0 & Referent & 0.005 \\
Absent & 0.3 & $(0.1-0.8)$ & \\
Punctate & 0.5 & $(0.3-0.8)$ & \\
Comedo & & & \\
Atypical ductal hyperplasia & Referent & \\
Absent & 1.0 & $(1.9-5.2)$ & \\
Present & 3.1 & & 0.0001 \\
Lobular neoplasia & & & \\
Absent & 1.0 & Referent & \\
Present & 2.3 & $(1.3-3.9)$ & \\
Columnar cell change & & & \\
Absent & 1.0 & Referent & \\
Present & 2.2 & $(1.3-3.7)$ & \\
\hline
\end{tabular}

${ }^{\mathrm{a}}$ Logistic model includes all variables in the table.

\section{Discussion}

Several prior small, observational studies have examined the relationship between flat epithelial atypia and histologic features of ductal carcinoma in situ.$^{3-14}$ However, our study represents the largest and most detailed study of this subject to date. In our review of histologic sections from 543 patients with ductal carcinoma in situ, we demonstrated that flat epithelial atypia is most often seen in association with ductal carcinoma in situ lesions with particular pathologic characteristics. The features of ductal carcinoma in situ most frequently associated with the presence of flat epithelial atypia in univariate analysis were low nuclear grade, and micropapillary and cribriform patterns. Furthermore, features such as comedo necrosis, stromal desmoplasia and stromal inflammation which are most often seen in association with high-grade ductal carcinoma in situ had an inverse association with the presence of flat epithelial atypia. In multivariable analysis, micropapillary and cribriform patterns and the absence of comedo necrosis were independently associated with the presence of flat epithelial atypia.

These findings are consistent with those of prior small observational studies indicating that flat epithelial atypia commonly coexists with welldeveloped examples of low-grade ductal carcinoma in situ and tubular carcinoma and that the cells comprising the flat epithelial atypia share cytologic and immunophenotypic features with the cells comprising these other lesions. ${ }^{3-14}$ Of interest, flat epithelial atypia was 3-times more common among ductal carcinoma in situ specimens that also showed atypical ductal hyperplasia than among those without atypical ductal hyperplasia. This observation provides further circumstantial evidence in support of a relationship between flat epithelial atypia, atypical ductal hyperplasia and low-grade ductal carcinoma in situ.

We also noted that flat epithelial atypia was seen more than twice as often among ductal carcinoma in situ specimens that also had lobular neoplasia (lobular carcinoma in situ and atypical lobular hyperplasia) than among those without lobular neoplasia. A number of authors have previously noted an association between flat epithelial atypia and lobular neoplasia. ${ }^{11,13,15}$ Recent genetic studies have suggested that lobular neoplasia and low-grade ductal neoplasia are closely related entities and our findings are consistent with those observations. ${ }^{19,20}$

A number of studies have investigated the genetic alterations in flat epithelial atypia. Moinfar et $a l^{12}$ showed loss of heterozygosity at one or more of the eight loci evaluated in 9 of 13 cases of 'Ductal Intraepithelial Neoplasia-flat monomorphic type' (flat epithelial atypia) and further, that the genetic alterations in these lesions were the same as those in the associated ductal carcinoma in situ or invasive cancer. In another study using comparative genomic hybridization to evaluate 81 lesions from 18 patients, Simpson et $a l^{16}$ found genomic changes not only in examples of flat epithelial atypia but also in examples of columnar cell change and columnar cell hyperplasia. In addition, in five of eight cases, there was overlap in the molecular profiles of the columnar cell lesions/flat epithelial atypia and coexistent ductal carcinoma in situ and invasive cancer, implying an evolutionary relationship. Additionally, Dabbs et $a{ }^{17}$ examined the spectrum of columnar cell lesions for loss of heterozygosity at 10 
loci. Losses were seen in 10 of 15 examples of atypical columnar cell hyperplasia/flat epithelial atypia. Again, some of the observed genetic alterations in the columnar cell lesions were similar to those seen in the associated ductal carcinoma in situ and invasive carcinoma suggesting a precursorproduct relationship. Most recently, Abdel-Fatah et $a l^{14}$ have demonstrated a very high prevalence of flat epithelial atypia in association with invasive carcinomas, particularly tubular and invasive lobular carcinomas, lending yet further support to the hypothesis that flat epithelial atypia represents a precursor along a low-grade neoplasia pathway.

Although the natural history of the flat epithelial atypia-ductal carcinoma in situ sequence can only be established in a prospective study, our observations, in conjunction with those of recent genetic studies, provide support for a precursor-product relationship among flat epithelial atypia, atypical ductal hyperplasia, low-grade ductal carcinoma in situ, and low-grade invasive breast cancers.

With regard to potential limitations to our study, although we found that $19 \%$ of the ductal carcinoma in situ subjects in this study showed associated flat epithelial atypia, it could be argued that this may not be representative of the prevalence of flat epithelial atypia in association with ductal carcinoma in situ in the general population, or in a larger cohort. The parent study from which our population was derived is a case-control study of women who had breast conserving therapy for the treatment of ductal carcinoma in situ. If flat epithelial atypia were to be associated with an increased risk for the development of recurrent ductal carcinoma in situ or progression to invasive cancer, its frequency would be expected to be higher among cases than it would be among controls or among a consecutive series of patients with ductal carcinoma in situ. However, given that the prevalence of flat epithelial atypia in association with ductal carcinoma in situ among cases and controls was similar, the fact that our study population was derived from a casecontrol study rather than a cohort study likely had little impact on our results and suggests that the prevalence of flat epithelial atypia in patients with ductal carcinoma in situ may, in fact, be approximately $20 \%$. However, the true prevalence of flat epithelial atypia in association with ductal carcinoma in situ would need to be determined from a prospective cohort study.

There are a number of strengths to our study worth emphasizing. First, in at least $82 \%$ of subjects all of the tissue submitted for pathologic examination was available for review (in some subjects the number of blocks submitted for pathologic examination was not recorded in the original pathology report). Second, all histologic sections were subject to central review by two pathologists with expertise in breast pathology. Finally, this analysis represents the largest population of women with ductal carcinoma in situ for which formal pathology review and systematic evaluation for the presence of flat epithelial atypia has been conducted.

In conclusion, we have demonstrated that flat epithelial atypia is significantly and independently associated with ductal carcinoma in situ with a micropapillary and/or cribriform architecture as well as an absence of comedo necrosis, features most often seen in low-grade ductal carcinoma in situ. While results of observational studies such as ours cannot be used to prove a precursor-product relationship between flat epithelial atypia and ductal carcinoma in situ with these features, our findings provide additional evidence to support the concept that flat epithelial atypia is an early event in the development of low-grade ductal neoplasia.

\section{Acknowledgement}

We thank the personnel in the pathology departments at Kaiser Permanente of Northern California, Kaiser Permanente of Southern California and Harvard Vanguard Medical Associates and affiliated hospitals. This study was supported by Public Health Service Grants U19CA079689 and R01CA081302 from the National Cancer Institute, National Institutes of Health, Department of Health and Human Services.

\section{Disclosure/conflict of interest}

None of these authors have personal financial interests or conflicts of interest to disclose.

\section{References}

1 Tavassoli FA, Hoefler H, Rosai J, et al. Intraductal proliferative lesions. In: Tavassoli FA, Devilee P (eds). Pathology and Genetics: Tumours of the Breast and Female Genital Organs. IARC Press: Lyon, 2003, pp 63-73.

2 Azzopardi JG. Problems in Breast Pathology. WB Saunders: Philadelphia, 1979.

3 Eusebi V, Feudale E, Foschini MP, et al. Long-term follow-up of in situ carcinoma of the breast. Semin Diagn Pathol 1994;11:223-235.

4 Fraser JL, Raza S, Chorny K, et al. Columnar alteration with prominent apical snouts and secretions: a spectrum of changes frequently present in breast biopsies performed for microcalcifications. Am J Surg Pathol 1998;22:1521-1527.

5 Oyama T, Iijima K, Takei H, et al. Atypical cystic lobule of the breast: an early stage of low-grade ductal carcinoma in-situ. Breast Cancer 2000;7:326-331.

6 Weidner N. Malignant breast lesions that may mimic benign tumors. Semin Diagn Pathol 1995;12:2-13.

7 Page DL KM, Jensen RA. Hypersecretory hyperplasia with atypia in breast biopsies. What is the proper level of clinical concern? Pathol Case Rev 1996;1:36-40.

8 Wellings SR, Jensen HM, Marcum RG. An atlas of subgross pathology of the human breast with special 
reference to possible precancerous lesions. J Natl Cancer Inst 1975;55:231-273.

9 Goldstein NS, O’Malley BA. Cancerization of small ectatic ducts of the breast by ductal carcinoma in situ cells with apocrine snouts: a lesion associated with tubular carcinoma. Am J Clin Pathol 1997;107: 561-566.

10 Rosen PP. Rosen's Breast Pathology, 2nd edn. Lippincott-Raven: Philadelphia, PA, 2001.

11 Rosen PP. Columnar cell hyperplasia is associated with lobular carcinoma in situ and tubular carcinoma. Am J Surg Pathol 1999;23:1561.

12 Moinfar F, Man YG, Bratthauer GL, et al. Genetic abnormalities in mammary ductal intraepithelial neoplasia-flat type ('clinging ductal carcinoma in situ'): a simulator of normal mammary epithelium. Cancer 2000;88:2072-2081.

13 Sahoo S, Recant WM. Triad of columnar cell alteration, lobular carcinoma in situ, and tubular carcinoma of the breast. Breast J 2005;11:140-142.

14 Abdel-Fatah TM, Powe DG, Hodi Z, et al. High frequency of coexistence of columnar cell lesions, lobular neoplasia, and low grade ductal carcinoma in situ with invasive tubular carcinoma and invasive lobular carcinoma. Am J Surg Pathol 2007;31:417-426.

15 Brogi E, Oyama T, Koerner FC. Atypical cystic lobules in patients with lobular neoplasia. Int J Surg Pathol 2001;9:201-206.

16 Simpson PT, Gale T, Reis-Filho JS, et al. Columnar cell lesions of the breast: the missing link in breast cancer progression? A morphological and molecular analysis. Am J Surg Pathol 2005;29:734-746.

17 Dabbs DJ, Carter G, Fudge M, et al. Molecular alterations in columnar cell lesions of the breast. Mod Pathol 2006;19:344-349.

18 Schnitt SJ, Collins LC. Columnar cell lesions and flat epithelial atypia of the breast. Semin Breast Dis 2005; 8:100-111.

19 Buerger H, Simon R, Schafer KL, et al. Genetic relation of lobular carcinoma in situ, ductal carcinoma in situ, and associated invasive carcinoma of the breast. Mol Pathol 2000;53:118-121.

20 Reis-Filho JS, Simpson PT, Gale T, et al. The molecular genetics of breast cancer: the contribution of comparative genomic hybridization. Pathol Res Pract 2005;201: $713-725$ 